RUNNING HEAD: SINGLE SESSION SERVICES

\title{
Association between single session service attendance and clinical characteristics in administrative data
}

Julian Edbrooke-Childs*, PhD, Daniel Hayes, PhD., Rebecca Lane, MSc., Shaun Liverpool, $\mathrm{PhD}$., Jenna Jacob, $\mathrm{PhD} \&$ Jessica Deighton, $\mathrm{PhD}$

Evidence Based Practice Unit, University College London \& Anna Freud National Centre for Children and Families, Clinical, Educational and Health Psychology, Gower Street, London, WC1E 6BT, UK

*Corresponding author. Email: EBPU@ annafreud.org, tel: +44 (0)20 77942313

\section{Authors' Financial Disclosures}

The authors report no biomedical financial interest or potential conflicts of interest.

\section{Acknowledgements}

The authors would like to thank all members of CORC, its Board at the time of writing including: Ashley Wyatt, Mick Atkinson, Kate Martin, Kate Dalzell, Ann York, Duncan Law, Isobel Fleming, Amy Mary Rose Herring, Rebecca Lewis and Beth Ingram - and the CORC team at the time of writing (including J.E-C and J.J.): Benjamin Richie, Luís Costa Da Silva, Meera Patel, Sally Marriott, Lee Atkins, Martha Reilly, Nicholas Tait, Anisatu Rashid, Rosie Singleton, Anja Teichert, Florence Ruby, and Kate Dalzell. The authors would also like to thank Miranda Wolpert for helpful comments on the analysis and previous drafts of the manuscript.

\section{Funding statement}


The authors did not receive specific funding for this research.

\title{
Data availability statement
}

The data that support the findings of this study are available from the Child Outcomes Research Consortium. Restrictions apply to the availability of these data, which were used under license for this study. Data are available

https://www.corc.uk.net/media/1883/request-for-use-of-corc-dataset-27-09-2018.doc with the permission of the Child Outcomes Research Consortium.

\begin{abstract}
A large proportion of young people accessing specialist mental health services do so for a single session. The aim of the present study was to examine the characteristics of young people attending specialist mental health services for a single session and to examine associations between single session attendance and clinical characteristics. Secondary analysis of administrative data on $N=23,300$ young people (mean age $=12.73$ years, $57 \%$ female, $64 \%$ White British) was conducted. The mean number of sessions attended was 4.33 and $46 \%$ $(10,669)$ attended for a single session. Multilevel logistic regression analysis showed that younger children, Black young people $(\mathrm{OR}=1.20,95 \% \mathrm{CI}=1.01-1.43)$ or those whose ethnicity was not stated $(\mathrm{OR}=1.25,95 \% \mathrm{CI}=1.15-1.35)$, young people with peer relationship difficulties $(\mathrm{OR}=1.11,95 \% \mathrm{CI}=1.04-1.19)$ or low frequency problems $(\mathrm{OR}=1.06,95 \% \mathrm{CI}$ $=0.99-1.14)$, and those with more complexity factors $(\mathrm{OR}=1.07,95 \% \mathrm{CI}=1.04-1.10)$ were more likely to attend services for a single session. The present study sets out research questions to prompt future research: 1) the experience of attending services for a single session, 2) identifying groups of single session attenders who do not require further support compared to those who are not able to sustain engagement with more sessions, 3) whether new care
\end{abstract}


pathways are needed for these groups who currently access specialist mental health services for a single session.

Keywords: youth mental health; single session attenders; clinical characteristics; service utilization 


\section{Association between single session service attendance and clinical characteristics in}

\section{administrative data}

Children and young people have the highest levels of mental health difficulties across the lifespan (Kessler et al., 2005). Despite this, access to mental health services has been found to be the lowest in children and adolescents across ages (McGorry et al., 2013). Low levels of treatment access have been repeatedly reported (Reardon et al., 2017), with a national survey in the United Kingdom (UK) reporting that $66 \%$ of young people with a mental health difficulty were in contact with professional services, though only $25 \%$ was through mental health specialists (Sadler et al., 2017). Research into factors influencing access to children and young people's mental health services (CYPMHS) has suggested that predictors of access include subjective perception of difficulties, service location, service affordability, and cultural sensitivity (Pandiani et al., 2005; Reardon et al., 2017). Furthermore, particular groups appear to have additional barriers to accessing services, with for example, children and young people from minority ethnic groups, or those in contact with welfare or youth justice services, having lower levels of service access (Pandiani et al., 2005; Stein et al., 2003).

A model which aims to understand how children and young people access services and seek help, the Gateway Provider Model (Stiffman et al., 2003), stipulates that a central role in young people's access to treatment is the individual who identifies a problem and refers to treatment (i.e., the gateway provider). It is suggested that the providers' perception of need, knowledge of resources, environment (Stiffman et al., 2001; 2003), their trust of services (Logan \& King, 2001), and the perceived stigma of mental health problems (Dempster et al., 2013) are predictors of the decision to refer to services. Parents/carers are often a primary gateway provider for young people, not only supporting access to appointments but also playing a critical role in ensuring adherence to treatment (Reardon et al., 2017). Furthermore, parents/carers often play a critical role in care and treatment, for example, as co-service-users, 
co-therapists, or the direct focus of the intervention (Creswell et al., 2013; Wolpert et al., 2005). There is an increased focus on utilization rates as a prime measure of access to care (Stiles et al., 2002). Nevertheless, access to care extends beyond the first point of access and includes factors pertaining to both treatment processes and treatment outcomes.

A study examining classification approaches to understanding young people's utilization of CYPMHS (Martin et al., 2017), found that the modal number of appointments attended was one, with $24 \%$ of all appointments being closed after the first appointment (Wolpert et al., 2015). When examined in terms of resource use, both presenting problem and severity of impairment independently predicted number of sessions up to case closure. Across ages, young people with emotional difficulties, where high impairment was also reported by the clinician, accessed a greater number of sessions than other young people presenting at services. However, young people aged six-to-twelve years presenting with conduct problems and autism were found to access a greater number of sessions. Conversely, young people aged 13 years or over presenting with psychosis or eating disorders were found to have accessed the greatest number of sessions across ages and problem types. Nevertheless, the authors noted that there was variation, both within presenting problems, but also within services (Martin et al., 2017). Research further afield in Canada has also demonstrated that CYPMHS resource use may be predicted by a positive association with family burden, child impairment and externalising difficulties (Reid et al., 2019). This research also found that those with low levels of these difficulties and contextual factors, who were seen for fewer sessions than those with higher levels of these difficulties, were also less likely to return to treatment within a four year time period, which suggests that those within this group do not have poorer outcomes than those seen for longer periods of time.

Building on this, clinical expertise informed the development of a classification system of 18 needs-based groups across three overarching categories: getting advice, getting help, and 
getting more help (Martin et al., 2017). Resource and service use within the getting advice group tended to be lower than the getting help and getting more help groups, but again there was considerable variation within this (Martin et al., 2017). These groupings have since been used to form the basis of the THRIVE model (Wolpert et al., 2015). THRIVE is an integrated and person-centered model for children, young people, and parents/carers focussed with addressing the needs of families at its heart (Wolpert et al., 2015). It conceptualizes need into five categories: Thriving, Getting Advice, Getting Help, Getting More Help, and Getting Risk Support. Each section of the model is unique in terms of :1) the needs and choices of patients, 2) the skill mix required by professionals who help and support young people, 3) the language used to describe need, and 4) the resources required to meet the needs and choices of patients. Based on this model, "getting advice" is the least resource intensive and may only attend for a single session. This may include those who have mild difficulties or who are adjusting to life circumstances, where support can be provided in the community, including within schools or self-support. In addition, it may also include individuals who have continuing difficulties, where a shared decision is made not to start treatment at this stage, and those who feel that selfhelp with such difficulties is sufficient. There is a need for additional research on this "getting advice" group of young people who access mental health services for only a single session to understand this group further.

To date, the long-term commitment of young people and families is considered to be a key element in successful interventions, with an emphasis on a failure to treat if non-attendance occurs after one session (Campbell, 1999). Research has however demonstrated that singlesession attenders do not have poorer outcomes than those who attend for more than one session (Bloom, 2001; Reid et al., 2019; Talmon, 1990). Further, with the high proportion of young people previously demonstrated as attending for a single session only (Wolpert et al., 2015), it is important to understand the specific needs of this group. This is particularly important in 
light of the prior research which highlights differential access to services amongst families (Pandiani et al., 2005; Reardon et al., 2017; Sadler et al., 2017; Stein et al., 2003) and differences in single session attenders between and within services (Martin et al., 2017; Reid et al., 2019). Given the paucity of research in this specific area, the present research is key to contribute towards beginning to understand who these young people are, which in turn will allow further consideration to be given to how to best support them and their families, and the associated resource allocation in models such as THRIVE. Patterns of single session attenders may well be reflective of challenges with engaging families in CYPMHS (De Haan et al., 2013; Gopalan et al., 2010), or such patterns and explorations may lead to the increased need for brief interventions. Such research is particularly important to inform future investigations unpacking reasons why children and young people might attend services for a single session as for some it may indeed reflect successfully meeting "getting advice" needs, while for others it may reflect premature termination, rapid changes in mental health or life circumstances meaning care is no longer needed or accessible, or a mis-match between perceived needs with service provision.

To address this aim, the present study examined administrative data to examine the characteristics of young people attending specialist mental health services for a single session, and to examine the association between single session attendance and clinical characteristics, to further understanding of this group and to prompt further investigation. The study will help develop understanding of the client group who accesses services for a single session and the important characteristics that need to be examined.

\section{Methods}

\section{Participants and procedure}


The data corpus was collected from child and adolescent mental health services participating in a programme to implement evidence-based practice in the UK between 2011 and 2015 across England (Jones et al., 2013). Services from least deprived to more deprived areas in England were involved, subsequently capturing data from rural and urban areas. Episodes of care were included in the present analysis if the young people were aged $\leq 25$ years (a widely established cut-off for statutory and non-statutory work with young people in the UK), had complete demographic characteristics (i.e., age, gender, ethnicity), and had attended at least one session or event. We also included only those with complete presenting problem and complexity factor information (see Measures), which was available for $40 \%$ of the sample, resulting in a final dataset of $N=23,300$ episodes of care $^{1}$ (mean (SD) age $=12.73(3.5)$ years, $57 \%$ female, $64 \%$ White British). Detailed demographic characteristics and descriptive information on all study variables are shown in Table 1 . These demographic characteristics are not necessarily representative of the wider population (UK Government, 2011). However, they are broadly representative of young people seen by CYPMHS, where research has shown an under-representation of certain groups, such as young people from Black or minoritized ethnic groups in CYPMHS (Edbrooke-Childs et al., 2016; Malek \& Joughlin, 2004). In the UK, provision for CYPMHS is free for service-users within the National Health Service (NHS).

[INSERT TABLE 1 HERE]

\section{Ethical considerations}

The present analysis involved secondary analysis of anonymized administrative data and therefore, ethical review was not required (NHS Health Research Authority, 2015).

\section{Demographic characteristics.}

Age, gender, and ethnicity were recorded by services as part of routine data recording. For the main analysis, age was coded as 0-12 years, 13-15 years, and 16+ years. Ethnicity was captured using the categories from the 2001 Census and based on youth-report and/or 
parent/carer-report. As we have used in previous research (Edbrooke-Childs \& Patalay, 2019) these were grouped for analysis as: White British (as the ethnic majority group), White Other (including Irish and Other White background), mixed-race (including Mixed White and Black Caribbean, Mixed White and Black African, Mixed White and Asian, and any other mixed background), Asian (including Indian, Pakistani, Bangladeshi, and Other), Black or Black British (including Caribbean, African, and Other), other ethnic groups (including Chinese and Other), and not stated.

\section{Measures.}

To measure case characteristics, 44 items of the Current View (CV; Jones et al. 2013) questionnaire were used which capture presenting problems and complexity factors. The CV questionnaire is a symptom checklist completed by therapists during an initial assessment appointment, with guidance and training available for scoring.

Presenting problems. To identify presenting problems, 30 items of the CV questionnaire was used. Clinicians rated the presence of 30 presenting problems, nevertheless we minimized the inclusion of under-powered groups in the main analysis by using 21 presenting problems and categorized those occurring with a frequency of $\leq 10 \%$ as "Other" problems (e.g., bipolar disorder, psychosis, substance abuse, elimination problems, selective mutism, gender identity, unexplained physical symptoms, self-care issues, unexplained developmental difficulties, and adjustment to health issues). Presenting problems were coded 1 for present (rated at least mild) or 0 for absent (no problems).

Complexity factors. Complexity factors were identified using 14 items of the CV questionnaire. Clinicians rated the extent to which young people were experiencing complex factors (e.g., contact with youth justice, in need of social care input) and the total number present was computed.

\section{Analytic strategy}


Multilevel logistic regressions were conducted in STATA 14. In Model 0 (null model) the variance explained in single session attendance at the service-level was examined and no predictors were added. The intraclass correlation coefficient was $27 \%$ indicating there was significant service-level variation and confirming that multilevel regression was the correct analytical approach. In Model 1, demographic characteristics were added: female, age coded 13-15 years and 16+ years (where 0-12 years was selected as the reference category to facilitate interpretation), and ethnicity (where the White British group was selected as the reference category as it was the largest group). In Model 2, presenting problems and grand-mean centred total number of complexity factors were added. The likelihood ratio test was used to compare successive models, which were significant, and all variables were therefore retained; in particular, Model 2 was significant compared to Model 1: $\chi 2(22)=183, p<.001$.

\section{Results}

Overall, the mean $(\mathrm{SD})$ number of sessions attended was 4.33 (7.19) (median $=2$, mode $=1$, range $1-184,95 \%$ confidence interval 4.24-4.42), and $46 \%(10,669)$ had attended for only a single session. The results of the final model (Model 2) are shown in Table 2. Girls $(\mathrm{OR}=$ $0.82,95 \% \mathrm{CI}=0.76-0.87)$ and older children were less likely to attend for only a single session. In terms of presenting problems, young people with separation anxiety, generalized anxiety, obsessive compulsive disorder, panic disorder, specific phobia, eating disorder, depression, or self-harm were less likely to attend for only a single session than young people without these presenting problems. In contrast, young people with peer relationship difficulties or low frequency problems were more likely to attend for only a single session than young people without these problems. In terms of ethnicity, Black young people $(\mathrm{OR}=1.20,95 \% \mathrm{CI}=1.01$ $1.43)$, or those whose ethnicity was "not stated" ( $\mathrm{OR}=1.25,95 \% \mathrm{CI}=1.15-1.35)$ were more likely to attend for only a single session than young people from other ethnicity groups. In terms of complexity factors, young people with more complexity factors were more likely to 
attend for only a single session than young people with fewer complexity factors $(\mathrm{OR}=1.07$, $95 \% \mathrm{CI}=1.04-1.10)$.

\section{[INSERT TABLE 2 HERE]}

\section{Discussion}

The aim of the present study was to describe the characteristics of young people attending specialist mental health services for a single session, and to examine the association between single session attendance and clinical characteristics, to further the understanding of this group and to prompt further investigation. Based on the available data, a larger number of young people attended services for only a single session $(10,669 ; 46 \%)$ compared to previous research (Martin et al., 2017). Boys, younger children, young people of Black ethnic origin and young people not reporting a self-identified ethnicity were more likely to attend services for only a single session. In terms of presenting problems, young people experiencing peer relationship problems and "other" problems (i.e. those with low frequency in the current sample) were more likely to attend a single session. In addition, young people with more complexity factors were more likely to access services for only a single session.

The first point of interest is that almost half of the sample attended services for only a single session. This is consistent with previous studies examining dropout rates. For example, studies in the UK have found dropout rates of between $30-40 \%$ in youth mental health services (Wolpert et al., 2012) and 58\% in adult mental health services (Gaglia et al., 2013). Moreover, similar dropout rates of $57 \%$ are reported in mental health services in other countries for young adults 18-32 years (Reneses et al., 2009). Notably, a direct comparison with other studies is not clear-cut, given that dropout is generally defined differently across studies (De Haan et al., 2013; Gaglia et al., 2013). Therefore, future research is needed to examine whether young people and families attending specialist mental health services for a single session should be considered as "dropouts" or whether a single session had been sufficient to meet their needs. There is research to support the latter, indicating improvements in both severity and frequency 
of problems after single session therapies (Boyhan, 1996; Perkins, 2006; Price, 1994). In this vein, the current findings can inform the THRIVE model of care. This is where young people and their families will access services based on need (e.g. a single session to obtain advice) and services could adapt and upskill practitioners to facilitate this.

The current findings also highlight demographic differences, suggesting that boys, younger children and young people from minority ethnic groups are more likely to attend a single session. One potential reason for this is that there are additional barriers from minority ethnic groups when accessing care, such as differences around attitudes towards services or language difficulties which make engagement more difficult (Pandiani et al., 2005; Stein et al., 2003), and adds to the existing knowledge on health inequalities in young people's mental health services (Simmons et al., 2011).

However, there may also be relationship considerations to take into account. The therapeutic alliance has been found to predict engagement, yet is affected by various influences, including clinician factors such as skills and knowledge (Karver et al., 2006). If clinicians are unable to connect with young people and families, it may be that single session attendance is due to a perceived lack of fit. Additionally, with the move to include young people in care and treatment decisions (Department of Health, 2015), clinicians may risk alienating either the young person or parent if consensus around ways forward cannot be reached, leading to disengagement. Skills such as containment, negotiation and cultural awareness are needed by clinicians to navigate these issues (Hayes et al., 2019) and may help mitigate single session attendance due to clinician factors.

Another important finding is that accessing services for a single session was predicted by the presence of less frequently occurring problems, as well as peer relationship problems. For peer relationship problems, single session attendance may be due to services being unable to provide further clinical input as the young person does not meet a certain threshold or care pathway. Current evidence-based approaches often focus on treatment for peer difficulties facilitated in educational or recreational settings (Fabiano et al., 2010). Thus, it may be that 
services refer on to practitioners in the community better able to facilitate treatment. Conversely, for low frequency problems, single session attendance is challenging to disentangle due to the heterogeneity in the range of problems incorporated. Some of these difficulties may be common in the experience of young people, if not commonly recorded in presentation at CAMHS, single session attendance in these instances may represent shifting perspectives in where support for such difficulties are best placed. Other low frequency problems may result in brief contact because of a lack of staff with specialist knowledge, rather than due to patients not meeting a clinical threshold or specific care pathway. As a result, these cases are referred on to those who are able to provide more specialist treatment, such as substance abuse centres.

Peer relationship problems, as well as low frequency problems such as substance abuse sometimes coexist with complexity factors such as learning difficulties and family relationship difficulties (Cantwell \& Baker, 1991; Denton \& Kampfe, 1994) further identifying additional barriers to accessing care. Previous research has demonstrated that children and young people with fewer difficulties and contextual issues specific to family burden, child impairment and externalising difficulties were more likely to require minimal input (Reid et al., 2019). This raises further questions about service utility and barriers to care. Conversely, young people with complex problems including separation anxiety, generalized anxiety, obsessive compulsive disorder, panic disorder, specific phobia, eating disorder, depression, or self-harm continuing to access services (i.e. more than a single session). While it is possible that extended contact associated with these conditions may reflect those that services are able to effectively engage with and support, extended contact does also point to greater need in these instances. For instance, despite the developments in home based and outpatient care, a number of young people require inpatient care. These families may then go on to seek more focused or extensive goals-based treatment as indicated by the TRIVE model. Taken together, these findings are 
crucial to inform service requirement, cost-effectiveness and accountability in the provision of publicly funded mental health services. Similarly, these findings may begin to help understand brief therapy and single session therapy session previously implemented out of necessity (Perkins, 2006). Ongoing outcome exploration of children and young people who attend CYPMHS for brief periods of treatment is required for further understanding of the sufficiency of meeting the required needs of this group and to determine whether all parties experience single sessions as beneficial.

Limitations of the present study include use of administrative data, meaning there may have been differences in how services collected and coded the data. The focus of the present research was on single session attendance; therefore, more detailed explorations of service engagement were not possible. Moreover, it is unclear whether these young people attended a single session because further support was not required or because they disengaged with further support. To begin to unpick these questions, the present study sets out four research questions to prompt future research and investigation. One, what are young people's and parents'/carers' experiences of attending services for a single session? Two, are there sub-groups within those attending single sessions, such as those needing only signposting support, those who perhaps found the session less helpful and chose not to continue, and those who were not able to sustain engagement with more sessions? Three, are new care pathways needed for these groups of young people and parents'/carers' who currently access specialist mental health services for a single session? And four, is there a need for services to be offered differently in order to engage some groups of service? Additionally, the high proportion of young people who attend for a single session may be linked to the overarching referral process, which it was not possible to ascertain from the present dataset. These referral routes and processes are likely to differ across countries and thus the results demonstrated here are likely to only be applicable to the country within which the data were collected, therefore further generalisation should be approached 
with caution. Notwithstanding the need for further research, some considerations might be offered for clinical practice based on the current findings. For instance, ensuring time for reflection during initial sessions to establish how the contact matched expectations and whether there are any initial concerns might be beneficial. Wider consideration of structural or institutional barriers that services may present to some groups of children and families also require extensive consideration.

In conclusion, a high percentage of young people discontinue service use after a single session attendance. This study highlights possible associations with some demographic and clinical characteristics raising further research questions. Therefore, we hope the present study will promote future empirical investigation of these important, unanswered questions. 


\section{Summary}

In this paper, we explored the characteristics of young people attending specialist mental health services for a single session and examined the association between single session attendance and clinical characteristics. We found that boys; younger children; Black young people or those whose ethnicity was not stated; young people with peer relationship difficulties, or low frequency problems; and young people with more complexity factors were more likely to attend services for only a single session. Future research is needed to examine the experience of attending services for a single session, to identify groups of single session attenders who do not require further support compared to those who are not able to sustain engagement with more sessions, and to explore whether new care pathways are needed for these groups of young people and families who currently access specialist mental health services for a single session.

\section{Footnotes}

${ }^{1}$ In the data corpus, pseudonymized data are uploaded according to episodes of care. Therefore, it is possible that a young person may have been included under more than one episode of care. 


\section{References}

Bloom, B. L. (2001). Focused Single-Session Psychotherapy: A Review of the Clinical and Research Literature. Brief Treatment \& Crisis Intervention, 1(1).

Boyhan, P. A. (1996). Clients' perceptions of single session consultations as an option to waiting for family therapy. Australian and New Zealand Journal of Family Therapy, 17(2), 85-96. Doi: 0.1002/j.1467-8438.1996.tb01078.x

Campbell, A. (1999). Single session interventions: An example of clinical research in practice. Australian and New Zealand Journal of Family Therapy, 20(4), 183-194. Doi: 10.1111/j.0814-723X.1999.00128.X

Cantwell, D. P., \& Baker, L. (1991). Association between attention deficit-hyperactivity disorder and learning disorders. Journal of learning disabilities, 24(2), 88-95. Doi: $\underline{10.1177 / 002221949102400205}$

Creswell, C., Cartwright-Hatton, S., \& Rodriguez, N. (2013). Working collaboratively with parents and carers in cognitive behaviour therapy (CBT). Cognitive Behaviour Therapy for Children and Families, 91-110.

De Haan, A. M., Boon, A. E., de Jong, J. T., Hoeve, M., \& Vermeiren, R. R. (2013). A metaanalytic review on treatment dropout in child and adolescent outpatient mental health care. Clinical psychology review, 33(5), 698-711. Doi: 10.1016/j.cpr.2013.04.005

Dempster, R., Wildman, B., \& Keating, A. (2013). The role of stigma in parental helpseeking for child behavior problems. Journal of Clinical Child \& Adolescent Psychology, 42(1), 56-67. Doi: 10.1080/15374416.2012.700504

Denton, R. E., \& Kampfe, C. M. (1994). The relationship between family variables and adolescent substance abuse: A literature review. Adolescence, 29(114), 475-495.

Department of Health. (2015). Future in mind: Promoting, protecting and improving our children and young people's mental health and wellbeing. https://assets.publishing.service.gov.uk/government/uploads/system/uploads/attachme nt_data/file/414024/Childrens_Mental_Health.pdf

Edbrooke-Childs, J., \& Patalay, P. (2019). Ethnic differences in referral routes to youth mental health services. Journal of the American Academy of Child \& Adolescent Psychiatry, 58(3), 368-375. Doi: 10.1016/j.jaac.2018.07.906

Edbrooke-Childs, J., Newman, R., Fleming, I., Deighton, J., \& Wolpert, M. (2016). The association between ethnicity and care pathway for children with emotional problems in routinely collected child and adolescent mental health services data. European Child \& Adolescent Psychiatry, 25(5), 539-546. Doi: 10.1007/s00787-015-0767-4

Fabiano G.A., Vujnovic R.K., Pariseau M.E. (2010) Peer Problems. In: Thomas J.C., Hersen M. (eds) Handbook of Clinical Psychology Competencies. Springer, New York, NY. Doi: 10.1007/978-0-387-09757-2_57

Gaglia, A., Essletzbichler, J., Barnicot, K., Bhatti, N., \& Priebe, S. (2013). Dropping out of dialectical behaviour therapy in the NHS: the role of care coordination. The Psychiatrist, 37(8), 267-271. Doi: 10.1192/pb.bp.112.041251 
Gopalan, G., Goldstein, L., Klingenstein, K., Sicher, C., Blake, C., \& McKay, M. M. (2010). Engaging families into child mental health treatment: Updates and special considerations. Journal of the Canadian Academy of Child and Adolescent Psychiatry / Journal de l'Académie canadienne de psychiatrie de l'enfant et de l'adolescent, 19(3), 182-196.

Hayes, D., Edbrooke-Childs, J., Town, R., Wolpert, M., \& Midgley, N. (2019). Barriers and facilitators to shared decision making in child and youth mental health: clinician perspectives using the theoretical domains framework. European child \& adolescent psychiatry, 28(5), 655-666. Doi: 0.1007/s00787-018-1230-0

Jones, M., Hopkins, K., Kyrke-Smith, R., Davies, R., Vostanis, P., \& Wolpert, M. Current view tool: Completion guide. 2013. London: CAMHS Press.

Logan, D. E., \& King, C. A. (2001). Parental facilitation of adolescent mental health service utilization: A conceptual and empirical review. Clinical Psychology: Science and Practice, 8(3), 319-333. Doi: 10.1093/clipsy.8.3.319

Karver, M. S., Handelsman, J. B., Fields, S., \& Bickman, L. (2006). Meta-analysis of therapeutic relationship variables in youth and family therapy: The evidence for different relationship variables in the child and adolescent treatment outcome literature. Clinical psychology review, 26(1), 50-65. Doi: 10.1016/j.cpr.2005.09.001

Kessler, R. C., Berglund, P., Demler, O., Jin, R., Merikangas, K. R., \& Walters, E. E. (2005). Lifetime prevalence and age-of-onset distributions of DSM-IV disorders in the National Comorbidity Survey Replication. Archives of general psychiatry, 62(6), 593602. doi:10.1001/archpsyc.62.6.593

Malek, H., \& Joughlin, C. (2004). Mental health services for minority ethnic children and adolescents. Jessica Kingsley Publishers.

Martin, P., Davies, R., Macdougall, A., Ritchie, B., Vostanis, P., Whale, A., \& Wolpert, M. (2017). Developing a case mix classification for child and adolescent mental health services: the influence of presenting problems, complexity factors and service providers on number of appointments. Journal of Mental Health, 29(4), 431-438. Doi: $\underline{10.1080 / 09638237.2017 .1370631}$

McGorry, P., Bates, T., \& Birchwood, M. (2013). Designing youth mental health services for the 21st century: examples from Australia, Ireland and the UK. The British Journal of Psychiatry, 202(s54), s30-s35. doi: 10.1192/bjp.bp.112.119214

NHS Health Research Authority. Governance Arrangements for Research Ethics Committees (GAfREC). 2015. http://www.hra.nhs.uk/resources/research-legislation-andgovernance/governance-arrangements-for-research-ethics-committees/. (Accessed 27/02/19).

O’Keeffe, S., Martin, P., Goodyer, I. M., Wilkinson, P., Consortium, I., \& Midgley, N. (2018). Predicting dropout in adolescents receiving therapy for depression. Psychotherapy Research, 28(5), 708-721. Doi: $\underline{10.1080 / 10503307.2017 .1393576}$

Pandiani, J.A., Banks, S.M., Simon, M.M., Van Vleck, M.C., \& Pomeroy, S.M. (2005). Access to child and adolescent mental health services. Journal of Child and Family Studies. 14(3), 431-41.

Perkins, R. (2006). The effectiveness of one session of therapy using a single-session therapy approach for children and adolescents with mental health problems. Psychology and 
Psychotherapy: Theory, Research and Practice, 79(2), 215-227. Doi: $\underline{10.1348 / 147608305 \times 60523}$

Price, C. (1994). Open days making family therapy accessible in working class suburbs. Australian and New Zealand Journal of Family Therapy, 15(4), 191-196. Doi: 10.1002/j.1467-8438.1994.tb01011.x

Reardon, T., Harvey, K., Baranowska, M., O’Brien, D., Smith, L., \& Creswell, C. (2017). What do parents perceive are the barriers and facilitators to accessing psychological treatment for mental health problems in children and adolescents? A systematic review of qualitative and quantitative studies. European child \& adolescent psychiatry, 26(6), 623-647. DOI 10.1007/s00787-016-0930-6

Reid, G. J., Stewart, S. L., Barwick, M., Carter, J., Leschied, A., Neufeld, R. W., ... \& Zaric, G. S. (2019). Predicting patterns of service utilization within children's mental health agencies. BMC health services research, 19(1), 993.

Reneses, B., Munoz, E., \& Lopez-Ibor, J. J. (2009). Factors predicting drop-out in community mental health centres. World Psychiatry, 8(3), 173-177. Doi: 10.1002/j.2051-5545.2009.tb00246.x

Sadler, K., Vizard, T., Ford, T., Marchesell, F., Pearce, N., Mandalia, D., Davis, J., Brodie, E., Forbes, N., Goodman, A. \& Goodman, R. (2018). Mental health of children and young people in England, 2017.

Shin, K. M., Cho, S. M., Shin, Y. M., \& Park, K. S. (2016). Effects of early childhood peer relationships on adolescent mental health: A 6-to 8-year follow-up study in South Korea. Psychiatry investigation, 13(4), 383-388. Doi: $10.4306 /$ pi.2016.13.4.383

Simmons, M. B., Hetrick, S. E., \& Jorm, A. F. (2011). Experiences of treatment decision making for young people diagnosed with depressive disorders: a qualitative study in primary care and specialist mental health settings. BMC psychiatry, 11(1), 194-206. Doi: 10.1186/1471-244X-11-194

Stein, S. M., Christie, D., Shah, R., Dabney, J., \& Wolpert, M. (2003). Attitudes to and knowledge of CAMHS: Differences between Pakistani and white British mothers. Child and Adolescent Mental Health, 8(1), 29-33. Doi: 10.1111/1475$\underline{3588.00042}$

Stiffman, A. R., Pescosolido, B., \& Cabassa, L. J. (2004). Building a model to understand youth service access: The gateway provider model. Mental health services research, 6(4), 189-198. Doi: 10.1023/B:MHSR.0000044745.09952.33

Stiffman, A. R., Striley, C., Horvath, V. E., Hadley-Ives, E., Polgar, M., Elze, D., \& Pescarino, R. (2001). Organizational context and provider perception as determinants of mental health service use. The Journal of Behavioral Health Services \& Research, 28(2), 188-204. Doi: 10.1007/BF02287461

Stiles, P. G., Boothroyd, R. A., Snyder, K., \& Zong, X. (2002). Service penetration by persons with severe mental illness: How should it be measured?. The Journal of Behavioral Health Services \& Research, 29(2), 198-207. Doi: 10.1007/BF02287706 
Talmon, M. (1990). Single-session therapy: Maximizing the effect of the first (and often only) therapeutic encounter. Jossey-Bass.

UK Government (2011). Ethnicity facts and figures. https://www.ethnicity-factsfigures.service.gov.uk/uk-population-by-ethnicity/demographics/agegroups/latest\#main-facts-and-figures (Accessed 01/02/2021).

Wolpert, M., Doe, J., \& Elsworth, J. (2005). Working with parents: some ethical and practical issues. Cognitive behaviour therapy for children and families, 103-120.

Wolpert, M., Harris, R., Hodges, S., Fuggle, P., James, R., Wiener, A., McKenna, C., Law, D., York, A., Jones, M. \& Fonagy, P. (2015). THRIVE elaborated. http://repository.tavistockandportman.ac.uk/1447/ (Accessed 30/09/20).

Wolpert, M., Jacob, J., Napoleone, E., Whale, A., Calderon, A., \& Edbrooke-Childs, J. (2016). Child- and Parent-reported Outcomes and Experience from Child and Young People's Mental Health Services 2011-2015. 2016. CAMHS Press.

Wolpert, M., Hoffman, J., Abrines, N., Feltham, A., Baird, L., Law, D., \& Hopkins, K. (2012). Closing the gap through changing relationships. The Health Foundation. 
Single session services 21 
Table 1.

Descriptive statistics for all study variables

$n, \%$ or mean $(S D)$

\section{Demographics}

Female

$0-12$ years

$13-15$ years

$16+$ years

White British

White other

Mixed-race

Asian

Black

Other

Not stated

Presenting problems and complexity factors

Separation anxiety

Social anxiety

Generalized anxiety

Obsessive compulsive disorder

Panic disorder

Agoraphobia

Specific phobia

Habit problems

Eating disorder

Depression

Self-harm

Hyperactivity

Behavioral difficulties

Poses risk to self and other

Carer management problems

Post-traumatic stress disorder

Family relationship difficulties

Peer relationship difficulties

Attachment problems

Emerging personality disorder

Low frequency problems

Mean (SD) total number of complexity factors

\section{Number of sessions}

Mean (SD) number of sessions

Single session
$13,212,57 \%$

$8,788,38 \%$

$9,313,40 \%$

$5,199,22 \%$

$14,857,64 \%$

$820,4 \%$

$861,4 \%$

$582,3 \%$

$850,4 \%$

$346,1 \%$

$4,984,21 \%$

$31 \%, 7,203$

$42 \%, 9815$

$44 \%, 10,266$

$18 \%, 4,164$

$25 \% 5,808$

$16 \%, 3,664$

$12 \%, 2,788$

$13 \%, 3,035$

$14 \%, 3,232$

$50 \%, 11,609$

$31 \%, 7,145$

$24 \%, 5,610$

$26 \%, 6,157$

$13 \%, 2,935$

$27 \%, 5,976$

$16 \%, 3,754$

$46 \%, 10,768$

$42 \%, 9,728$

$25 \%, 5,789$

$12 \%, 2,885$

$30 \%, 6,889$

$0.73(1.23)$

$4.33(7.19)$

$46 \%, 10,669$

Note. $N=23,300$. 
Table 2.

Multilevel regressions with demographic characteristics and presenting problems and complexity factors predicting single session attendance.

\begin{tabular}{llll}
\hline & OR & $95 \%$ CI & \\
\hline Demographics & & & \\
Female vs. male & $\mathbf{0 . 8 2}$ & 0.76 & 0.87 \\
13-15 years vs. 0-12 years & $\mathbf{0 . 7 4}$ & 0.68 & 0.79 \\
16+ vs. 0-12 years & $\mathbf{0 . 7 6}$ & 0.70 & 0.83 \\
White other vs. White British & 1.13 & 0.96 & 1.34 \\
Mixed-race & 0.90 & 0.77 & 1.06 \\
Asian & 1.17 & 0.95 & 1.42 \\
Black & $\mathbf{1 . 2 0}$ & 1.01 & 1.43 \\
Other & 1.02 & 0.79 & 1.32 \\
Not stated & $\mathbf{1 . 2 5}$ & 1.15 & 1.35 \\
Presenting problems and complexity factors & & & \\
Separation anxiety & $\mathbf{0 . 9 2}$ & 0.85 & 0.99 \\
Social anxiety & 0.99 & 0.92 & 1.08 \\
Generalized anxiety & $\mathbf{0 . 8 5}$ & 0.78 & 0.92 \\
Obsessive compulsive disorder & $\mathbf{0 . 8 8}$ & 0.80 & 0.96 \\
Panic disorder & $\mathbf{0 . 8 0}$ & 0.74 & 0.87 \\
Agoraphobia & 1.04 & 0.95 & 1.14 \\
Specific phobia & $\mathbf{0 . 8 9}$ & 0.80 & 0.98 \\
Habit problems & 1.03 & 0.94 & 1.14 \\
Eating disorder & $\mathbf{0 . 8 6}$ & 0.79 & 0.94 \\
Depression & $\mathbf{0 . 7 5}$ & 0.70 & 0.81 \\
Self-harm & $\mathbf{0 . 9 2}$ & 0.85 & 0.99 \\
Hyperactivity & 1.02 & 0.94 & 1.11 \\
Behavioral difficulties & 1.09 & 1.00 & 1.18 \\
Poses risk to self and other & 1.03 & 0.93 & 1.14 \\
Carer management problems & 0.96 & 0.88 & 1.04 \\
Post-traumatic stress disorder & 0.96 & 0.88 & 1.04 \\
Family relationship difficulties & 1.01 & 0.94 & 1.09 \\
Peer relationship difficulties & $\mathbf{1 . 1 1}$ & 1.04 & 1.19 \\
Attachment problems & 0.94 & 0.87 & 1.02 \\
Emerging personality disorder & 1.04 & 0.94 & 1.14 \\
Low frequency problems & $\mathbf{1 . 0 6}$ & 0.99 & 1.14 \\
Total number of complexity factors & $\mathbf{1 . 0 7}$ & 1.04 & 1.10 \\
\hline Note. N=23,300. OR & & \\
\hline
\end{tabular}

Note. $N=23,300 . \mathrm{OR}=$ odds ratio. $\mathrm{CI}=$ confidence interval. Coefficients in bold are significant at least at the $p<.05$ level. 